\title{
A New Plethysm Formula for Symmetric Functions
}

\author{
WILLIAM F. DORAN IV \\ Department of Mathematics, California Institute of Technology, Pasadena, CA 91125
}

Received December 29, 1995; Revised June 2, 1997

Abstract. This paper gives a new formula for the plethysm of power-sum symmetric functions and Schur symmetric functions with one part. The form of the main result is that for $\mu \vdash b$,

$$
p_{\mu}(\underline{x}) \circ s_{a}(\underline{x})=\sum_{T} \underline{\omega}^{\operatorname{maj}_{\mu}(T)} s_{\mathrm{sh}(T)}(\underline{x})
$$

where the sum is over semistandard tableaux $T$ of weight $a^{b}, \underline{\omega}$ is a root of unity, and $\operatorname{maj}_{\mu}(T)$ is a major index like statistic on semistandard tableaux.

An $S_{b}$-representation, denoted $S^{\lambda, b}$, is defined. In the special case when $\lambda \vdash b, S^{\lambda, b}$ is the Specht module corresponding to $\lambda$. It is shown that the character of $S^{\lambda, b}$ on elements of cycle type $\mu$ is

$$
\sum_{T} \underline{\omega}^{\operatorname{maj}_{\mu}(T)}
$$

where the sum is over semistandard tableaux $T$ of shape $\lambda$ and weight $a^{b}$. Moreover, the eigenvalues of the action of an element of cycle type $\mu$ acting on $S^{\lambda, b}$ are $\left\{\underline{\omega}^{\operatorname{maj}_{\mu}(T)}: T\right\}$. This generalizes J. Stembridge's result [11] on the eigenvalues of elements of the symmetric group acting on the Specht modules.

Keywords: symmetric function, plethysm, eigenvalue, representation of the symmetric group

\section{Introduction}

\subsection{Tableaux}

A partition of $n$ is a weakly decreasing sequence $\lambda=\left(\lambda_{1} \geq \cdots \geq \lambda_{l}\right)$ of positive integers which sum to $n$. Both $|\lambda|=n$ and $\lambda \vdash n$ is used to denote that $\lambda$ is a partition of $n$. The value $l$ is the number of parts of $\lambda$ and is denoted $l(\lambda)$. Let $[\lambda]=\{(i, j): 1 \leq i \leq l(\lambda)$ and $1 \leq$ $\left.j \leq \lambda_{i}\right\} \subset \mathbf{Z}^{2}$. The set $[\lambda]$ is the Ferrers diagram of $\lambda$ and is thought of as a collection of boxes arranged using matrix coordinates. The conjugate of $\lambda$ is the partition $\lambda^{\prime}$ whose Ferrers diagram $\left[\lambda^{\prime}\right]$ is the transpose of $[\lambda]$.

A tableau of shape $\lambda$ and weight (or content) $\alpha=\left(\alpha_{1}, \ldots, \alpha_{k}\right)$ is a filling of the Ferrers diagram of $\lambda$ with positive integers such that $i$ appears $\alpha_{i}$ times. A tableau is semistandard if its entries are weakly increasing from left to right in each row and strictly increasing down each column. In this paper, the primary class of tableaux of interest is semistandard tableaux of weight $(b, \ldots, b)$ which is abbreviated $b^{a}$. If $\lambda \vdash a b$, let $\mathcal{S}^{\lambda, a}$ be the set of semistandard $a$ times 
tableaux of shape $\lambda$ and weight $b^{a}$ and $\mathcal{W}^{\lambda, a}$ be the set of tableaux of shape $\lambda$ and weight $b^{a}$. Tableaux of weight $1^{n}$ are called standard.

If $[\nu] \subseteq[\lambda]$, let $[\lambda / \nu]$ denote the skew-shape $[\lambda] \backslash[\nu]$. A filling of $[\lambda / \nu]$ with $\alpha_{i}$ many $i$ 's is a skew-tableau of shape $\lambda / \nu$ and weight $\alpha$. A semistandard skew-tableau is defined similarly.

\subsection{Symmetric functions}

The symmetric function notation in this papers closely follows that of Chapter 1 in Macdonald [9]. Let $\Lambda^{n}$ denote the ring of symmetric functions of homogeneous degree $n$ with rational coefficients in the variables $\left\{x_{1}, x_{2}, \ldots\right\}$. Let $\Lambda=\bigoplus_{n \geq 0} \Lambda^{n}$ be the ring of symmetric functions. Two important bases of $\Lambda$ both of which are indexed by partitions are the Schur symmetric functions $s_{\lambda}(\underline{x})$ and the power sum symmetric functions $p_{\lambda}(\underline{x})$. $\Lambda$ has a bilinear, symmetric, positive definite scalar product given by $\left\langle s_{\lambda}, s_{\mu}\right\rangle=\delta_{\lambda, \mu}$.

When two Schur symmetric functions are multiplied together and expanded in terms of Schur symmetric functions,

$$
s_{\mu}(\underline{x}) s_{\nu}(\underline{x})=\sum_{\lambda \vdash|\mu|+|v|} c_{\mu, \nu}^{\lambda} s_{\lambda}(\underline{x}),
$$

the resulting multiplication coefficients $c_{\mu, v}^{\lambda}$ are nonnegative integers. These coefficients are called Littlewood-Richardson coefficients. See either Section I.9 of [9] or Section 4.9 of [10] for details.

Let $f(\underline{x})$ and $g(\underline{x})$ be symmetric functions. The plethysm of $f(\underline{x})$ and $g(\underline{x})$ is denoted $f(\underline{x}) \circ g(\underline{x})$. Since plethysm results in this paper are proven via a result of A. Lascoux, B. Leclerc, and J.-Y. Thibon [6], a definition of plethysm is omitted. The key results of [6] are reviewed in Section 4. A definition of plethysm is given in Section I.8 of [9]. Plethysm is not symmetric. However, it does have the property of being algebraic in the first coordinate. A proof of this proposition is given in Section I.8 of [9].

\section{Proposition 1.1}

(a) $\left(f_{1}(\underline{x})+f_{2}(\underline{x})\right) \circ g(\underline{x})=\left(f_{1}(\underline{x}) \circ g(\underline{x})\right)+\left(f_{2}(\underline{x}) \circ g(\underline{x})\right)$.

(b) $\left(f_{1}(\underline{x}) f_{2}(\underline{x})\right) \circ g(\underline{x})=\left(f_{1}(\underline{x}) \circ g(\underline{x})\right)\left(f_{2}(\underline{x}) \circ g(\underline{x})\right)$.

When taking the plethysm of two Schur symmetric functions,

$$
s_{\mu}(\underline{x}) \circ s_{\nu}(\underline{x})=\sum_{\lambda \vdash|\mu||\nu|} a_{\mu, \nu}^{\lambda} s_{\lambda}(\underline{x})
$$

the resulting plethysm coefficients $a_{\mu, \nu}^{\lambda}$ are nonnegative. However, no good combinatorial description of these numbers is known. The main result of this paper is a new formula for $p_{\mu}(\underline{x}) \circ s_{a}(\underline{x})$. Using Proposition 1.1, this gives a method for computing $f(\underline{x}) \circ s_{a}(\underline{x})$ for any symmetric function $f(\underline{x})$. 


\subsection{Characters of $S_{n}$}

Let $S_{n}$ denote the symmetric group on $n$ objects. The conjugacy class of a permutation is determined by its cycle type. Thus, the conjugacy classes of $S_{n}$ are indexed by partitions of $n$. For $\lambda \vdash n$, let $z_{\lambda}=\prod_{i \geq 1} i^{n_{i}(\lambda)} n_{i}(\lambda)$ ! where $n_{i}(\lambda)$ equals the number of parts of $\lambda$ equal to $i$. The number of permutations in $S_{n}$ with cycle type $\lambda$ is $n ! / z_{\lambda}$.

Let $R^{n}$ be the vector space of rational valued class functions on $S_{n}$. If $f \in R^{n}$ and $\lambda \vdash n$, $f(\lambda)$ is used to denote the value of $f$ on permutations of cycle type $\lambda$. $R^{n}$ has a bilinear, symmetric, positive definite scalar product given by

$$
\langle f, g\rangle=\frac{1}{n !} \sum_{\sigma \in S_{n}} f(\sigma) g\left(\sigma^{-1}\right)=\sum_{\lambda \vdash n} \frac{1}{z_{\lambda}} f(\lambda) g(\lambda) .
$$

In the above formula, the fact that $\sigma$ and $\sigma^{-1}$ have the same cycle type is used. For $\lambda \vdash n$, denote the irreducible character of $S_{n}$ corresponding to $\lambda$ by $\chi^{\lambda}$. The set $\left\{\chi^{\lambda}: \lambda \vdash n\right\}$ is an orthonormal (with respect to the just defined scalar product) basis for $R^{n}$. Another basis for $R^{n}$ is given by $\left\{\phi^{\lambda}: \lambda \vdash n\right\}$ where $\phi^{\lambda}(\mu)=\delta_{\lambda, \mu}$.

Let $R=\bigoplus_{n \geq 0} R^{n}$. Define the characteristic map ch: $R \rightarrow \Lambda$ by $\operatorname{ch}: \phi^{\lambda} \mapsto \frac{1}{z_{\lambda}} p_{\lambda}(\underline{x})$. The next proposition list several facts about the characteristic map which are used in this paper. Using these results, the characteristic map converts symmetric function results into results about $S_{n}$-characters and vice-versa. This is done without explicit mention. Proofs are given in Section I.7 of [9].

\section{Proposition 1.2}

(a) ch is a vector space isomorphism between $\Lambda$ and $R$.

(b) ch is an isometry (i.e., $\left.\langle f, g\rangle_{R}=\langle\operatorname{ch}(f), \operatorname{ch}(g)\rangle_{\Lambda}\right)$.

(c) $\operatorname{ch}\left(\chi^{\lambda}\right)=s_{\lambda}(\underline{x})$.

The following proposition list some useful facts about symmetric functions and their relationship with $S_{n}$-characters. Proofs are given in Chapter I of [9].

\section{Proposition 1.3}

(a) $s_{\lambda}(\underline{x})=\sum_{\mu \vdash|\lambda|}\left(\chi^{\lambda}(\mu) / z_{\mu}\right) p_{\mu}(\underline{x})$.

(b) $p_{\mu}(\underline{x})=\sum_{\lambda \vdash|\mu|} \chi^{\lambda}(\mu) s_{\lambda}(\underline{x})$.

(c) $\left\langle p_{\lambda}(\underline{x}), p_{\mu}(\underline{x})\right\rangle=\delta_{\lambda, \mu} z_{\lambda}$.

\section{Formula for $p_{\mu}(\underline{x}) \circ s_{a}(\underline{x})$}

Definition Given a semistandard tableau $T, i$ is a descent with multiplicity $k$ if there exists $k$ disjoint pairs $\left\{\left(x_{1}, y_{1}\right), \ldots,\left(x_{k}, y_{k}\right)\right\}$ of boxes in the Ferrers diagram of $T$ such that the entry in each $x_{j}$ is $i$, the entry in each $y_{j}$ is $i+1, y_{j}$ is in a lower row than $x_{j}$ for all $j$, and there does not exist a set of $k+1$ pairs of boxes which satisfy these conditions. Let $m_{i}(T)$ denote the multiplicity of $i$ as a descent in $T$. 
Example 1 Let $T$ be the following semistandard tableau.

$\begin{array}{lllllll}1 & 1 & \underline{1} & \underline{1} & \underline{2} & \underline{2} & \underline{4}\end{array}$

$2 \underline{2} \quad \underline{3} \quad 4$

$\begin{array}{llll}3 & 3 & 4 & 5\end{array}$

In this example, $m_{1}(T)=2, m_{2}(T)=3, m_{3}(T)=1, m_{4}(T)=1$. The positions of the $x_{j}$ 's which contribute to descent statistic are underlined.

One method for selecting the $\left(x_{j}, y_{j}\right)$ pairs which contribute to $m_{i}(T)$ is the following.

(i) Set $j=0$.

(ii) Let $x$ be the right-most $i$ which has not been previously considered.

(iii) Let $y$ be the right-most $i+1$ which is to left of or directly below $x$ and has not already been selected as a $y_{j}$.

(iv) If such a $y$ exists, increment $j$ by one, let $x_{j}=x$, let $y_{j}=y$, and add $\left(x_{j}, y_{j}\right)$ to the list of pairs.

(v) If there are any $i$ which have not been considered, goto step (ii). Otherwise, stop.

The statistic $m_{i}(T)$ equals the number of pairs found. This algorithm clearly generates a list of $\left(x_{j}, y_{j}\right)$ pairs which satisfy the definition of descent. In the above example, the underlined $x_{j}$ are the ones obtained by this algorithm. Step (ii) systematically goes through the $i$ 's from right to left. What happens if the order in which the $i$ 's are considered is changed? Surprisingly, the size of the list of $\left(x_{j}, y_{j}\right)$ pairs does not depend on the order in which the $i$ are considered as possible $x$ 's so long as the choice for the corresponding $y$ is the "greedy" choice of the right-most allowable $i+1$ as in step (iii). In fact as a set, the resulting $i+1$ 's which make up the $y_{j}$ 's do not depend on the order in which the $i$ 's are examined. Thus, to compute $m_{i}(T)$, the $i$ 's may be consider in any order. This is proven after the next example and plays a key role later in Lemma 3.1.

Example 2 Suppose $i=3$ and the relevant part of $T$ is

$$
\begin{array}{llllll} 
& & & 3_{2} & 3_{1} & 4_{1} \\
& 3_{4} & 3_{3} & 4_{2} & & \\
4_{4} & 43 & & & &
\end{array}
$$

The subscripts differentiate the various 3's and 4's and increase from right to left. Consider the pairs of 3's and 4's where the 3 is in a higher row than the 4 (or equivalently the 3 is in the same column or a column to right of the 4 ). The dots in the picture below indicate the possible pairs.

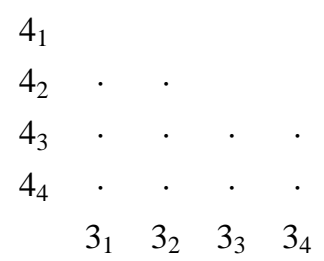


The above algorithm for selecting $\left(x_{j}, y_{j}\right)$ pairs is to work through the columns from left to right, and in each column select the highest row which has not already been selected and contains a dot in that column. The $X$ 's in the pictures below indicate the selections for two possible ordering of columns. Notice that the rows containing an $X$ are the same in both.

$\begin{array}{ccccccccccc}4_{1} & & & & & 4_{1} & & & & \\ 4_{2} & X & . & & & 4_{2} & & X & & . \\ 4_{3} & \cdot & X & . & . & 4_{3} & X & . & . & . \\ 4_{4} & \cdot & \cdot & X & . & 4_{4} & . & . & X & . \\ & 3_{1} & 3_{2} & 3_{3} & 3_{4} & & & 3_{4} & 3_{1} & 3_{3} & 3_{2}\end{array}$

Theorem 2.1 When selecting $\left(x_{j}, y_{j}\right)$ pairs by the above greedy algorithm, the set of $y_{j}$ 's selected does not depend on the order in which the i's are considered.

Proof: Suppose two orderings of the $i$ 's differ by a neighboring transposition. Since the symmetric group is generated by neighboring transpositions, it suffices to prove that the rows selected do not change under this transposition. Following the notation of Example 2, suppose columns $k$ and $k+1$ are transposed. Assume without loss of generality that the number of dots in column $k$ is greater than or equal to the number in column $k+1$. The rows selected while considering columns 1 to $k-1$ are the same in both. Suppose in column $k$ that row $y^{\prime}$ is selected and in column $k+1$ row $y^{\prime \prime}$ is selected. Since the number of dots in column $k$ is greater than the number of dots in column $k+1$, row $y^{\prime}$ is higher than row $y^{\prime \prime}$. When columns $k$ and $k+1$ are transposed, there are two cases based on whether or not there is dot in column $k+1$ row $y^{\prime}$. If there is a dot in this position, then in the transposed case, in column $k+1$, row $y^{\prime}$ is selected and in column $k$, row $y^{\prime \prime}$ is selected. If there is no dot in this position, then in the transposed case, in column $k+1$, row $y^{\prime \prime}$ is selected and in column $k$, row $y^{\prime}$ is selected. In either case, rows selected by columns $k$ and $k+1$ are the same. Thus, no differences occur in the later selections.

Definition Given a semistandard tableau $T$, for $j, k \geq 1$, define the $(j, j+k)$-major index, denoted $\operatorname{maj}_{j, j+k}(T)$, by

$$
\operatorname{maj}_{j, j+k}(T)=\sum_{i=j}^{j+k-1}(i-j+1) m_{i}(T) .
$$

Define $\operatorname{maj}_{j, j}(T)$ to be 0 . 
Example 3 Using (1) as $T$.

$$
\begin{aligned}
& \operatorname{maj}_{1,2}(T)=1 \cdot 2 \quad=2 \\
& \operatorname{maj}_{1,3}(T)=1 \cdot 2+2 \cdot 3=8 \\
& \operatorname{maj}_{1,4}(T)=1 \cdot 2+2 \cdot 3+3 \cdot 1=11 \\
& \operatorname{maj}_{1,5}(T)=1 \cdot 2+2 \cdot 3+3 \cdot 1+4 \cdot 1=15 \\
& \operatorname{maj}_{2,3}(T)=1 \cdot 3=3 \\
& \operatorname{maj}_{2,4}(T)=1 \cdot 3+2 \cdot 1=5 \\
& \operatorname{maj}_{2,5}(T)=1 \cdot 3+2 \cdot 1+3 \cdot 1=8 \\
& \operatorname{maj}_{3,4}(T)=1 \cdot 1=1 \\
& \operatorname{maj}_{3,5}(T)=\quad 1 \cdot 1+2 \cdot 1=3 \\
& \operatorname{maj}_{4,5}(T)=\quad 1 \cdot 1=1
\end{aligned}
$$

When $T$ is standard, $\operatorname{maj}_{1, n}(T)$ is the usual major index of a standard tableau. As a slight abuse of notation, let maj $(T)$ denote $\operatorname{maj}_{1, n}(T)$, where $n$ is the largest entry appearing in $T$, even when $T$ is not standard. The statistic maj $j_{j, j+k}(T)$ can be viewed as $\operatorname{maj}\left(T^{\prime}\right)$ where $T^{\prime}$ is the semistandard skew-tableau formed by the entries $\{j, \ldots, j+k\}$ in $T$, but with each entry reduced by $j-1$ so that the values which appear in $T^{\prime}$ run from 1 to $k$.

Definition Given a partition $\mu \vdash b$ of length $l$, let $r_{i}=\mu_{1}+\cdots+\mu_{i}$. Set $r_{0}=0$. Given a semistandard tableau $T$ with entries less than or equal to $b$. Define

$$
\underline{\omega}^{\operatorname{maj}_{\mu}(T)}=\prod_{i=1}^{l} \omega_{\mu_{i}}^{\operatorname{maj}_{r_{i-1}, r_{i}}(T)}
$$

where $\omega_{\mu_{i}}=e^{2 \pi i / \mu_{i}}$.

Example 4 Let $T$ be (1). The value of $\underline{\omega}^{\text {maj }_{\mu}}(T)$ is computed for every $\mu \vdash 5$.

$\begin{array}{lll}\mu & \underline{\omega}^{\text {maj }_{\mu}(T)} & \\ (5) & \omega_{5}^{15} & =1 \\ (4,1) & \omega_{4}^{11} \omega_{1}^{0} & =-i \\ (3,2) & \omega_{3}^{8} \omega_{2}^{1} & =-\omega_{3}^{2} \\ (3,1,1) & \omega_{3}^{8} \omega_{1}^{0} \omega_{1}^{0} & =\omega_{3}^{2} \\ (2,2,1) & \omega_{2}^{2} \omega_{2}^{1} \omega_{1}^{0} & =-1 \\ (2,1,1,1) & \omega_{2}^{2} \omega_{1}^{0} \omega_{1}^{0} \omega_{1}^{0} & =1 \\ (1,1,1,1,1) & \omega_{1}^{0} \omega_{1}^{0} \omega_{1}^{0} \omega_{1}^{0} \omega_{1}^{0}=1\end{array}$

Some more examples are done in Appendix A. Now the main result can be stated. 
Theorem 2.2 Let $\mu \vdash b$, then

$$
p_{\mu}(\underline{x}) \circ s_{a}(\underline{x})=\sum_{\substack{\text { SST } T \\ \operatorname{wt}(T)=a^{b}}} \underline{\omega}^{\text {maj }_{\mu}(T)} s_{\operatorname{sh}(T)}(\underline{x}) .
$$

Example 5 Using the values found in Appendix A,

$$
\begin{aligned}
p_{(1,1,1)} & \circ s_{(2)}=s_{(6)}+2 s_{(5,1)}+3 s_{(4,2)}+s_{(4,1,1)}+s_{(3,3)}+2 s_{(3,2,1)}+s_{(2,2,2)}, \\
p_{(2,1)} & \circ s_{(2)}=s_{(6)}+s_{(4,2)}-s_{(4,1,1)}-s_{(3,3)}+s_{(2,2,2)}, \\
p_{(3)} & \circ s_{(2)}=s_{(6)}-s_{(5,1)}+s_{(4,1,1)}+s_{(3,3)}-s_{(3,2,1)}+s_{(2,2,2)} .
\end{aligned}
$$

The " $(\underline{x})$ "s have been omitted.

Corollary 2.3 Let $\mu \vdash b$, then

$$
\left\langle p_{\mu}(\underline{x}) \circ s_{a}(\underline{x}), s_{\lambda}(\underline{x})\right\rangle=\sum_{\substack{\operatorname{SST} T \\ \operatorname{sh}(T)=\lambda, \operatorname{wt}(T)=a^{b}}} \underline{\omega}^{\operatorname{maj}_{\mu}(T)} .
$$

A similar but different formula for $p_{\mu}(\underline{x}) \circ s_{a}(\underline{x})$ is given in [2]. Their work is also reproduced in Example 8 of Section I.8 of [9].

\section{Charge and Kostka polynomials}

In this section, the charge of a semistandard tableau is defined and is related to the major index. Most of the definitions in this section are taken from Chapter 2 of [1] which is an excellent reference on this material. It should be noted that these definitions are the "reverse" of those given in Section III.6 of [9]. However, they give the same value for the charge of a semistandard tableau.

Definition Let $T$ be a semistandard tableau or semistandard skew-tableau. The word of $T$, denoted $w(T)$, is the sequence of integers gotten by reading the entries of $T$ from left to right in each row starting with the bottom row and moving up.

Example 6 Let $T$ be (1). Then $w(T)=334522341111224$.

Definition Let $T$ be a semistandard tableau or semistandard skew-tableau of weight $1^{b}$. Assign an index to each number in $w(T)$ as follows: the number 1 is given index 0 , and if $i$ has index $r$, then $i+1$ is given index $r$ or $r+1$ depending on whether $i+1$ is to the left or right, respectively, of $i$ in $w(T)$. The charge of $T$, denoted c $(T)$, is the sum of these indices. 
Example 7 Let $T$ be

$\begin{array}{ccccc}1 & 2 & 6 & 7 & 12 \\ 3 & 5 & 8 & 9 & \\ 4 & 10 & 11 & & \end{array}$

Then

$$
w(T)=\begin{array}{cccccccccccc}
4 & 10 & 11 & 3 & 5 & 8 & 9 & 1 & 2 & 6 & 7 & 12 \\
1 & 5 & 6 & 1 & 2 & 4 & 5 & 0 & 1 & 3 & 4 & 7
\end{array}
$$

The index of each number has been written below it. Thus, $\mathrm{c}(T)=1+5+6+1+2+$ $4+5+0+1+3+4+7=39$.

Notice that the $i$ 's for which $i$ occurs to the right of $i+1$ in $w(T)$ are the descents of $T$. Thus, $\operatorname{maj}(T)$ is the sum of the $i$ 's such that $i$ is to the right of $i+1$ in $w(T)$. The definition of the major index can be extended to arbitrary words $w$ of weight $1^{b}$ by setting maj $(w)$ to be the sum of the $i$ 's such that $i$ is to the right of $i+1$ in $w$. Likewise, the definition of charge can be extended to arbitrary words of weight $1^{b}$ by the obvious generalization of the above definition of charge. So, for a standard tableau $T, \mathrm{c}(T)=\mathrm{c}(w(T))$ and $\operatorname{maj}(T)=\operatorname{maj}(w(T))$.

Proposition 3.1 Let $w$ be a word of weight $1^{b}$. Then

$$
c(w) \equiv\left\{\begin{array}{ll}
\operatorname{maj}(w)+\frac{b}{2} & \text { if } b \text { is even, } \\
\operatorname{maj}(w) & \text { if } b \text { is odd }
\end{array} \quad(\bmod b)\right.
$$

Proof: Let $D=\{i: i$ is to the right of $i+1\}$. So, maj $(w)=\sum_{i \in D} i$. When $i \in D, i$ and $i+1$ are assigned the same index when calculating $\mathrm{c}(w)$. When $i \notin D$, the index given to $i+1$ is one greater than that given to $i$. Thus, when $i \notin D$, the index of every $j>i$ is incremented by one and contributes $b-i$ to the charge of $w$.

$$
\begin{aligned}
c(w) & =\sum_{i \notin D} b-i \\
& =\sum_{i=1}^{b-1}(b-i)-\sum_{i \in D}(b-i) \\
& =\frac{b(b-1)}{2}-|D| b+\operatorname{maj}(w) \\
& \equiv \frac{b(b-1)}{2}+\operatorname{maj}(w)(\bmod b)
\end{aligned}
$$

When $b$ is even $\frac{b(b-1)}{2} \equiv \frac{b}{2}(\bmod b)$. When $b$ is odd, $\frac{b(b-1)}{2} \equiv 0(\bmod b)$.

The definition of charge is extended to semistandard tableau and semistandard skewtableau of arbitrary weight $\mu$ by decomposing $w(T)$ into several standard words $w_{1}, \ldots, w_{\mu_{1}}$ 
and defining the charge of $T$ be the sum of the charges of the $w_{i}$ 's. Let $w(T)$ be a word with weight $\mu$. To construct subword $w_{1}$, read $w(T)$ from right to left. Select the first 1 which occurs, then select the first 2 which occurs to the left of the previously selected 1 , and so on. If at any stage there is no $i+1$ to the left of $i$, then circle around to the right and search for $i+1$ again reading $w(T)$ from right to left. Continue until an $i$ is reached for which $i+1$ does not appear in $w(T)$. The word $w_{1}$ is formed by taking the selected numbers in the order in which they appear in $w(T)$. To construct $w_{2}$, remove the selected numbers which form $w_{1}$ from $w(T)$ and repeat the above process. Each subsequent $w_{i}$ is obtained by removing the numbers which make up $w_{i-1}$ from what remains of $w(T)$ and repeating the selection process. The weight of $w_{i}$ is $1^{\mu_{i}^{\prime}}$.

Definition Let $T$ be a semistandard tableau or semistandard skew-tableau of weight $\mu$. Let $w_{1}, \ldots, w_{\mu_{1}}$ be the decomposition of $w(T)$. The charge of $T$ is defined to be $\mathrm{c}\left(w_{1}\right)+$ $\cdots+\mathrm{c}\left(w_{\mu_{1}}\right)$.

\section{Example 8 Let $T$ be}

$\begin{array}{lllll}1 & 1 & 1 & 2 & 3 \\ 2 & 2 & 3 & 4 & 4 \\ 3 & 4 & & & \end{array}$

$w(T)=342234411123, w_{1}=3241, w_{2}=2413, w_{3}=4312, \mathrm{c}(T)=1+2+3=6$.

Definition Let $|\lambda|=|\mu|$. The Kostka polynomial, denoted $K_{\lambda, \mu}(q)$, is given by

$$
K_{\lambda, \mu}(q)=\sum_{\substack{\operatorname{SST} T \\ \operatorname{sh}(T)=\lambda, \operatorname{wt}(T)=\mu}} q^{\mathrm{c}(T)}
$$

This is not the usual definition of the Kostka polynomial. The fact that this is equivalent to the usual definition is a deep result of A. Lascoux and M. Schützenberger [8].

Lemma 3.2 Let $T$ be a semistandard tableau or semistandard skew-tableau of weight $a^{b}$, then

$$
\mathrm{c}(T) \equiv \begin{cases}\operatorname{maj}(T)+\frac{b}{2} & \begin{array}{l}
\text { if } b \text { is even and } a \text { is odd, } \\
\text { otherwise. }
\end{array}(\bmod b)\end{cases}
$$

Proof: Let $w_{1}, \ldots, w_{a}$ be the decomposition of $w(T)$. Each $w_{j}$ is a word of weight $1^{b}$. Let $k_{i}$ be the number of $w_{j}$ 's in which $i$ is to right of $i+1$. If $i$ is to right of $i+1$ in $w_{j}$, then $i+1$ is in a lower row than is $i$ in $T$. Each $i$ appears in one and only one $w_{j}$. Thus, the $w_{j}$ 's provide an ordering on the $i$ 's. The method for selecting $i+1$ in each word is exactly the "greedy" method described in step (ii) of the algorithm given in Section 2. Thus by 
Theorem 2.1, $k_{i}=m_{i}(T)$. So, $\operatorname{maj}(T)=\operatorname{maj}\left(w_{1}\right)+\cdots+\operatorname{maj}\left(w_{a}\right)$. By Proposition 3.1,

$$
\begin{aligned}
\operatorname{maj}(T) & \equiv\left\{\begin{array}{ll}
\left(\operatorname{maj}\left(w_{1}\right)+\frac{b}{2}\right)+\cdots+\left(\operatorname{maj}\left(w_{a}\right)+\frac{b}{2}\right) & \text { if } b \text { is even, } \\
\operatorname{maj}\left(w_{1}\right)+\cdots+\operatorname{maj}\left(w_{a}\right) & \text { if } a \text { is odd. }
\end{array} \quad(\bmod b)\right. \\
& \equiv\left\{\begin{array}{ll}
\operatorname{maj}(w)+a \frac{b}{2} & \text { if } b \text { is even, } \\
\operatorname{maj}(w) & \text { if } a \text { is odd. }
\end{array}(\bmod b)\right. \\
& \equiv\left\{\begin{array}{ll}
\operatorname{maj}(w)+\frac{b}{2} & \text { if } b \text { is even and } a \text { is odd, } \\
\operatorname{maj}(w) & \text { otherwise. }
\end{array} \quad(\bmod b)\right.
\end{aligned}
$$

Corollary 3.3 Let $\lambda \vdash a b$. Then

$$
K_{\lambda, a^{b}}\left(\omega_{b}\right)=(-1)^{(b-1) a} \sum_{\substack{\operatorname{SST} T \\ \operatorname{sh}(T)=\lambda, \operatorname{wt}(T)=a^{b}}} \omega_{b}^{\operatorname{maj}(T)} .
$$

Proof: By Lemma 3.1,

$$
\begin{aligned}
\omega_{b}^{\mathrm{c}(T)} & = \begin{cases}\omega_{b}^{\operatorname{maj}(T)+\frac{b}{2}} & \text { if } b \text { is even and } a \text { is odd } \\
\omega_{b}^{\operatorname{maj}(T)} & \text { otherwise. }\end{cases} \\
& = \begin{cases}-\omega_{b}^{\operatorname{maj}(T)} & \text { if } b \text { is even and } a \text { is odd, } \\
\omega_{b}^{\operatorname{maj}(T)} & \text { otherwise. }\end{cases} \\
& =(-1)^{(b-1) a} \omega_{b}^{\operatorname{maj}(T)}
\end{aligned}
$$

Thus, $K_{\lambda, a^{b}}\left(\omega_{b}\right)=\sum_{T} \omega_{b}^{\mathrm{c}(T)}=(-1)^{(b-1) a} \sum_{T} \omega_{b}^{\operatorname{maj}(T)}$.

Definition Let $|\mu|+|\nu|=|\lambda|$. The skew-Kostka polynomial denoted $K_{\lambda / \nu, \mu}(q)$, is given by

$$
K_{\lambda / \nu, \mu}(q)=\sum_{\substack{\operatorname{SSST} T \\ \operatorname{sh}(T)=\lambda / \nu, \operatorname{wt}(T)=\mu}} q^{\mathrm{c}(T)} .
$$

Since Lemma 3.2 holds for semistandard skew-tableaux as well as semistandard tableaux, the obvious analog of Corollary 3.3 with $\lambda$ replaced by $\lambda / v$ also holds. The next result gives the relationship between skew-Kostka polynomials and Kostka polynomials. A proof of this is given in Chapter 2 of [1].

Theorem 3.4 Let $|\mu|+|\nu|=|\lambda|$. Then

$$
K_{\lambda / v, \mu}(q)=\sum_{\eta \vdash|\mu|} c_{v, \eta}^{\lambda} K_{\eta, \mu}(q) .
$$


Corollary 3.5 Let $|\lambda|-|\nu|=a b$. Then

$$
\sum_{\substack{\operatorname{SSST} T \\ \operatorname{sh}(T)=\lambda / \nu, \operatorname{wt}(T)=a^{b}}} \omega_{b}^{\operatorname{maj}(T)}=\sum_{\eta \vdash a b}\left(c_{\nu, \eta}^{\lambda} \sum_{\substack{\operatorname{SST} T \\ \operatorname{sh}(T)=\eta, \operatorname{wt}(T)=a^{b}}} \omega_{b}^{\operatorname{maj}(T)}\right) .
$$

\section{Proof:}

$$
\begin{aligned}
& \sum_{\substack{\operatorname{SSST} T \\
\operatorname{sh}(T)=\lambda / \nu, \operatorname{wt}(T)=a^{b}}} \omega_{b}^{\operatorname{maj}(T)}=(-1)^{(b-1) a} \sum_{\substack{\operatorname{SSST} T \\
\operatorname{sh}(T)=\lambda / \nu, \operatorname{wt}(T)=a^{b}}} \omega_{b}^{\mathrm{c}(T)} \\
& =(-1)^{(b-1) a} K_{\lambda / v, a^{b}}\left(\omega_{b}\right) \\
& =(-1)^{(b-1) a} \sum_{\eta \vdash a b} c_{v, \eta}^{\lambda} K_{\eta, a^{b}}\left(\omega_{b}\right) \\
& =\sum_{\eta \vdash a b}\left(c_{v, \eta}^{\lambda} \sum_{\substack{\operatorname{SST} T \\
\operatorname{sh}(T)=\eta, \operatorname{wt}(T)=a^{b}}} \omega_{b}^{\operatorname{maj}(T)}\right)
\end{aligned}
$$

\section{Modified Hall-Littlewood functions}

This section contains a result of Lascoux, Leclerc, and Thibon [6] which ties the plethysm of power-sum symmetric functions and Schur symmetric functions to Kostka polynomials evaluated at roots of unity.

Definition Let $|\mu|=|\nu|$. Green's polynomial, $X_{\mu}^{v}(q)$, is given by

$$
X_{\mu}^{v}(q)=\sum_{\lambda \vdash|v|} \chi^{\lambda}(\mu) K_{\lambda, v}(q)
$$

Definition The modified Hall-Littlewood function, $Q_{v}^{\prime}(\underline{x} ; q)$ is given by

$$
\left\langle Q_{\nu}^{\prime}(\underline{x} ; q), p_{\mu}(\underline{x})\right\rangle=X_{\mu}^{\nu}(q) .
$$

\section{Theorem $4.1[6]$}

$$
Q_{a^{b}}^{\prime}\left(\underline{x} ; \omega_{b}\right)=(-1)^{(b-1) a} p_{b}(\underline{x}) \circ s_{a}(\underline{x}) .
$$




\section{Proof of the main result}

Proof of Theorem 2.2: Proof by induction on the number of parts of $\mu$. First suppose $\mu$ has one part. So $\mu=(b)$. Let $n=a b$ and $\lambda \vdash n$.

$$
\begin{aligned}
& \left\langle p_{b} \circ s_{a}(\underline{x}), s_{\lambda}(\underline{x})\right\rangle \\
& =(-1)^{(b-1) a}\left\langle Q_{a^{b}}^{\prime}\left(\underline{x}, \omega_{b}\right), s_{\lambda}(\underline{x})\right\rangle \quad(\text { Theorem 4.1) } \\
& =(-1)^{(b-1) a} \sum_{\mu \vdash n}\left\langle Q_{a^{b}}^{\prime}\left(\underline{x}, \omega_{b}\right),\left(\chi^{\lambda}(\mu) / z_{\mu}\right) p_{\mu}(\underline{x})\right\rangle \quad \text { (Proposition 1.3(a)) } \\
& =(-1)^{(b-1) a} \sum_{\mu \vdash n}\left(\chi^{\lambda}(\mu) / z_{\mu}\right) X_{\mu}^{a^{b}}\left(\omega_{b}\right) \\
& =(-1)^{(b-1) a} \sum_{\mu \vdash n}\left(\chi^{\lambda}(\mu) / z_{\mu}\right) \sum_{\eta \vdash n} \chi^{\eta}(\mu) K_{\eta, a^{b}}\left(\omega_{b}\right) \\
& =(-1)^{(b-1) a} \sum_{\eta \vdash n} K_{\eta, a^{b}}\left(\omega_{b}\right) \underbrace{\left(\sum_{\mu \vdash n} \chi^{\lambda}(\mu) \chi^{\eta}(\mu) / z_{\mu}\right)}_{=\delta_{\lambda, \eta}} \\
& =(-1)^{(b-1) a} K_{\lambda, a^{b}}\left(\omega_{b}\right) \\
& =\sum_{\substack{\operatorname{SST} T \\
\operatorname{sh}(T)=\lambda, \operatorname{wt}(T)=a^{b}}} \omega_{b}^{\operatorname{maj}_{(b)}(T)} \quad \text { (Corollary 3.3) }
\end{aligned}
$$

Since the Schur functions are a basis for the symmetric functions, this shows that

$$
p_{b} \circ s_{a}(\underline{x})=\sum_{\substack{\text { SST } T \\ \operatorname{wt}(T)=a^{b}}} \omega_{b}^{\operatorname{maj}_{(b)}(T)} s_{\operatorname{sh}(T)}
$$

which is the base case for the induction.

Let $l=l(\mu)$. Let $\mu^{*}$ be the partition $\left(\mu_{1}, \ldots, \mu_{l-1}\right)$. By the induction hypothesis, the theorem holds for $p_{\mu^{*}}(\underline{x}) \circ s_{a}(\underline{x})$ and $p_{\mu_{l}}(\underline{x}) \circ s_{a}(\underline{x})$.

$$
\begin{aligned}
& p_{\mu}(\underline{x}) \circ s_{a}(\underline{x}) \\
& =\left(p_{\mu^{*}}(\underline{x}) \circ s_{a}(\underline{x})\right)\left(p_{\mu_{l}}(\underline{x}) \circ s_{a}(\underline{x})\right) \\
& =\left(\sum_{\substack{\operatorname{SST} T_{1} \\
\operatorname{wt}\left(T_{1}\right)=a^{\left(b-\mu_{l}\right)}}} \underline{\omega}^{\operatorname{maj}_{\mu^{*}}\left(T_{1}\right)} s_{\mathrm{Sh}\left(T_{1}\right)}(\underline{x})\right)\left(\sum_{\substack{\operatorname{SST} T_{2} \\
\operatorname{wt}\left(T_{2}\right)=a^{\mu_{l}}}} \omega_{b}^{\operatorname{maj}_{(b)}\left(T_{2}\right)} s_{\operatorname{sh}\left(T_{2}\right)}\right) \\
& =\sum_{\substack{\operatorname{SST} T_{1} \\
\operatorname{wt}\left(T_{1}\right)=a^{\left(b-\mu_{l}\right)}}} \sum_{\substack{\operatorname{SST} T_{2} \\
\operatorname{wt}\left(T_{2}\right)=a^{\mu_{l}}}} \underline{\omega}^{\operatorname{maj}_{\mu^{*}}\left(T_{1}\right)} \omega_{\mu_{l}}^{\operatorname{maj}\left(T_{2}\right)} s_{\operatorname{Sh}\left(T_{1}\right)}(\underline{x}) s_{\operatorname{sh}\left(T_{2}\right)}(\underline{x})
\end{aligned}
$$




$$
\begin{aligned}
& \left\langle p_{\mu}(\underline{x}) \circ s_{a}(\underline{x}), s_{\lambda}\right\rangle \\
& =\sum_{\substack{\operatorname{SST} T_{1} \\
\operatorname{wt}\left(T_{1}\right)=a^{\left(b-\mu_{l}\right)}}} \sum_{\substack{\operatorname{SST} T_{2} \\
\operatorname{wt}\left(T_{2}\right)=a^{\mu_{l}}}} \underline{\omega}^{\operatorname{maj}_{\mu^{*}}\left(T_{1}\right)} \omega_{\mu_{l}}^{\operatorname{maj}\left(T_{2}\right)} c_{\operatorname{sh}\left(T_{1}\right), \operatorname{sh}\left(T_{2}\right)}^{\lambda} \\
& =\sum_{\nu \vdash a\left(b-\mu_{l}\right)} \sum_{\eta \vdash a \mu_{l}} \sum_{\substack{\operatorname{SST} T_{1} \\
\operatorname{wt}\left(T_{1}\right)=a^{\left(b-\mu_{l}\right)}, \operatorname{sh}\left(T_{1}\right)=\nu}} \sum_{\substack{\operatorname{SST} T_{2} \\
\operatorname{wt}\left(T_{2}\right)=a^{\mu l}, \operatorname{sh}\left(T_{2}\right)=\eta}} \underline{\omega}^{\operatorname{maj}_{\mu^{*}}\left(T_{1}\right)} \omega_{\mu_{l}}^{\operatorname{maj}\left(T_{2}\right)} c_{\nu, \eta}^{\lambda} \\
& =\sum_{\nu \vdash a\left(b-\mu_{l}\right)}\left(\sum_{\substack{\operatorname{SST} T_{1} \\
\operatorname{wt}\left(T_{1}\right)=a^{\left(b-\mu_{l}\right)}, \operatorname{sh}\left(T_{1}\right)=\nu}} \underline{\omega}^{\operatorname{maj}_{\mu^{*}}\left(T_{1}\right)}\right)\left(\sum_{\substack{\operatorname{SSST} T_{3} \\
\operatorname{wt}\left(T_{3}\right)=a^{\mu l}, \operatorname{sh}\left(T_{3}\right)=\lambda / \nu}} \omega_{\mu_{l}}^{\operatorname{maj}\left(T_{3}\right)}\right) \\
& =\sum_{\substack{\operatorname{SST} T_{4} \\
\operatorname{wt}\left(T_{4}\right)=a^{b}, \operatorname{sh}\left(T_{4}\right)=\lambda}} \underline{\omega}^{\operatorname{maj}_{\mu}\left(T_{4}\right)}
\end{aligned}
$$

In the last step, the following bijection $\varphi$ between $\cup_{v}\left\{\left(T_{1}, T_{3}\right): \operatorname{sh}\left(T_{1}\right)=v\right.$, wt $\left(T_{1}\right)=$ $\left.a^{b-\mu_{l}}, \operatorname{sh}\left(T_{3}\right)=\lambda / \mu, \operatorname{wt}\left(T_{3}\right)=a^{\mu_{l}}\right\}$ and $\left\{T_{4}: \operatorname{sh}\left(T_{4}\right)=\lambda, \operatorname{wt}\left(T_{4}\right)=a^{b}\right\}$. Construct $\varphi\left(T_{1}, T_{3}\right)$ by incrementing every entry of $T_{3}$ by $b-\mu_{l}$ and placing $T_{1}$ inside $T_{3}$. For example,

$$
\varphi\left(\begin{array}{ccccccc}
1 & 1 & 2 & & & & 1 \\
2 & & & & 1 & 2 & \\
& & & & &
\end{array}\right)=\begin{array}{llll}
1 & 1 & 2 & 3 \\
2 & 3 & 4 & \\
4 & & &
\end{array} .
$$

This is clearly a bijection. Furthermore, it has the property that

$$
\underline{\omega}^{\operatorname{maj}_{\mu^{*}}\left(T_{1}\right)} \omega_{\mu_{l}}^{\operatorname{maj}\left(T_{3}\right)}=\underline{\omega}^{\text {maj }_{\mu} \varphi\left(T_{1}, T_{3}\right)} .
$$

Again since the Schur symmetric functions form a basis, this computation gives the desired result.

Let $\alpha=\left(\alpha_{1}, \ldots, \alpha_{l}\right)$ be a composition of $b$ which when sorted is $\mu$. Define $\underline{\omega}^{\operatorname{maj}_{\alpha}(T)}$ in the analogous manner. Then since nothing in the above proof depended on the fact that $\mu$ is a partition, we have

$$
\prod_{i=1}^{l} p_{\alpha_{i}}(\underline{x}) \circ s_{a}(\underline{x})=\sum_{\substack{\operatorname{SST} T \\ \operatorname{sh}(T)=\lambda, \operatorname{wt}(T)=a^{b}}} \underline{\omega}^{\operatorname{maj}_{\alpha}(T)} .
$$

Since $p_{i}(\underline{x})$ 's commute and plethysm is algebraic in the first coordinate, the following result is proven. 
Corollary 5.1 Let $\alpha=\left(\alpha_{1}, \ldots, \alpha_{l}\right)$ be a composition of $b$ which when sorted is the partition $\mu$. Then

$$
p_{\mu}(\underline{x}) \circ s_{a}(\underline{x})=\sum_{\substack{\operatorname{SST} T \\ \operatorname{sh}(T)=\lambda, \operatorname{wt}(T)=a^{b}}} \underline{\omega}^{\text {maj }_{\alpha}(T)} .
$$

\section{Definition of $S^{\lambda, b}$}

By looking at the charts in Appendix A, one quickly guesses that for a fixed $\lambda \vdash a b$,

$$
\psi(\mu)=\sum_{\substack{\operatorname{SST} T \\ \operatorname{sh}(T)=\lambda, \operatorname{wt}(T)=a^{b}}} \underline{\omega}^{\text {maj }_{\mu}(T)}
$$

is a character of $S_{b}$. This section defines the representation $S^{\lambda, b}$ whose character is $\psi(\mu)$. In the next section, the even stronger result that the $\underline{\omega}^{\text {maj }_{\mu}(T)}$ as $T$ varies are the eigenvalues of an element of cycle type $\mu$ acting on $S^{\lambda, b}$ is proven.

Let $\lambda \vdash n=a b$. The definition of the $S_{b}$-representation $S^{\lambda, b}$ closely follows that of the Specht modules. The Specht modules are a concrete construction of the irreducible representations of $S_{b}$. When $a=1$ (so $\lambda \vdash b$ ), $S^{\lambda, b}$ is the Specht module corresponding to $\lambda$. James' monograph [4] and Sagan's book [10] are a good sources on the Specht modules.

There are two group actions on $\mathcal{W}^{\lambda, b}$ which are needed to define $S^{\lambda, b}$. The first is the action of $S_{b}$ by permuting the values of the entries. This action is denoted by $\pi \cdot T$.

\section{Example 9}

$$
(123) \cdot \begin{array}{llll}
2 & 1 & 3 & 1 \\
3 & 2 & & =
\end{array}=\begin{array}{llll}
3 & 2 & 1 & 2 \\
1 & 3 & &
\end{array}
$$

The second is the action of $S_{n}$ by permuting positions. This action is denoted by $\sigma * T$. For a given tableau $T$, let $R_{T}$ denote the subgroup of $S_{n}$ which set-wise fixes the rows of $T$, and let $C_{T}$ denote the subgroup of $S_{n}$ which set-wise fixes the columns of $T$. If the shape of $T$ is $\lambda$, and $\lambda^{\prime}$ is the conjugate partition of $\lambda$, then $R_{T} \simeq S_{\lambda_{1}} \times S_{\lambda_{2}} \times \cdots \times S_{\lambda_{l}}$ and $C_{T} \simeq S_{\lambda_{1}^{\prime}} \times S_{\lambda_{2}^{\prime}} \times \cdots \times S_{\lambda_{\lambda_{1}^{\prime}}^{\prime}}$.

\section{Example 10}

$$
\begin{aligned}
& \left\{\begin{array}{rrrr}
2 & 1 & 3 & 1 \\
\sigma * & & & \\
3 & 2 & &
\end{array}\right.
\end{aligned}
$$

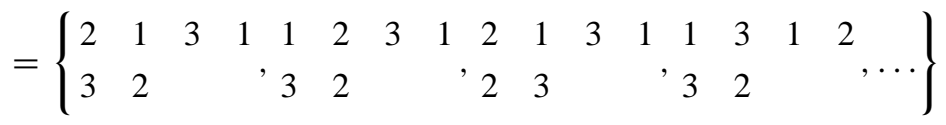

$$
\begin{aligned}
& \left\{\begin{array}{rrrr}
2 & 1 & 3 & 1 \\
3 & 2 & & \\
& & &
\end{array} \in C_{T}\right\}
\end{aligned}
$$

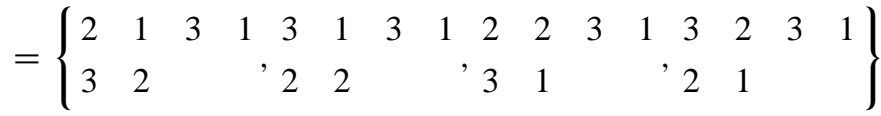


It should be noted that these two actions commute. That is, $\pi \cdot(\sigma * T)=\sigma *(\pi \cdot T)$.

Definition Let $W^{\lambda, b}$ be the complex vector space with basis $\mathcal{W}^{\lambda, b}$.

Since $S_{b}$ acts on $\mathcal{W}^{\lambda, b}, W^{\lambda, b}$ is a $S_{b}$-(permutation) representation.

Definition Given $T \in \mathcal{W}^{\lambda, b}$, define the element $e_{T}$ in $W^{\lambda, b}$ by

$$
e_{T}=\sum_{\sigma \in R_{T}} \sum_{\tau \in C_{T}}(\operatorname{sgn} \tau)(\sigma \tau) * T
$$

Let $S^{\lambda, b}$ be the subspace of $W^{\lambda, b}$ generated by $\left\{e_{T}: T \in \mathcal{W}^{\lambda, b}\right\}$.

Theorem 6.1 $S^{\lambda, b}$ is a subrepresentation of $W^{\lambda, b}$ with dimension $\left|\mathcal{S}^{\lambda, b}\right|$. Furthermore, $\left\{e_{T}: T \in \mathcal{S}^{\lambda, b}\right\}$ is a basis for $S^{\lambda, b}$.

As mentioned before, when $a=1, S^{\lambda, b}$ is the Specht module corresponding to $\lambda$. Since the proof of this Theorem follows the Specht module case so closely, the proof is omitted. See Chapter 4 of [4] or Section 2.3 of [10] for details. Let $\chi^{\lambda, b}$ be the character of $S^{\lambda, b}$. When $a=1$ (so $\lambda \vdash b$ ), $\chi^{\lambda, b}=\chi^{\lambda}$. The next result, in conjunction with Corollary 2.3, shows that $S^{\lambda, b}$ is the desired $S_{b}$-representation. A proof is given in [3].

Theorem 6.2 [3] Let $\lambda \vdash n=a b$ and $\mu \vdash b$. Then $\chi^{\lambda, b}(\mu)=\left\langle p_{\mu}(\underline{x}) \circ s_{a}(\underline{x}), s_{\lambda}\right\rangle$.

\section{Eigenvalues of $S^{\lambda, b}$}

The next result is the basic tool used to determine the eigenvalues of a group element acting on a representation. A proof is given in [11].

Proposition 7.1 Let $V$ be a representation of $G$ of dimension $n$ with character $\chi_{V}$. Let $g \in G$ be an element of order $m .\left\{\omega_{m}^{e_{1}}, \ldots, \omega_{m}^{e_{n}}\right\}$ are the eigenvalues of $g$ acting on $V$ if and only if

$$
\omega_{m}^{k e_{1}}+\cdots+\omega_{m}^{k e_{n}}=\chi_{V}\left(g^{k}\right)
$$

for all $0 \leq k<m$.

Lemma 7.2 Let $\lambda=a b$ and $d \mid b$. If $\zeta_{1}$ and $\zeta_{2}$ are both primitive $d$ th roots of unity, then $K_{\lambda, a^{b}}\left(\zeta_{1}\right)=K_{\lambda, a^{b}}\left(\zeta_{2}\right)$.

A proof of this is given in [6]. 
Corollary 7.3 Let $|\lambda / \nu|=n=a b$ and $d \mid b$. If $\zeta_{1}$ and $\zeta_{2}$ are both primitive $d$ th roots of unity, then

$$
\sum_{\substack{\operatorname{SSST} T \\ \operatorname{sh}(T)=\lambda / \nu, \operatorname{wt}(T)=a^{b}}} \zeta_{1}^{\operatorname{maj}(T)}=\sum_{\substack{\operatorname{SSST} T \\ \operatorname{sh}(T)=\lambda / \nu, \operatorname{wt}(T)=a^{b}}} \zeta_{2}^{\operatorname{maj}(T)} .
$$

Proof: This follows from Lemma 7.2 and the skew version of Corollary 3.3 referred to in the comments after the definition of skew-Kostka polynomials.

Lemma 7.4 Let $|\lambda / \nu|=n=a b$ and $d \mid b$. Then

$$
\begin{aligned}
& \sum_{\substack{\operatorname{SSST} T \\
\operatorname{sh}(T)=\lambda / \nu, \operatorname{wt}(T)=a^{b}}} \omega_{b}^{d \operatorname{maj}(T)} \\
= & \sum_{\substack{\nu=\eta_{0} \subset \eta_{1} \subset \cdots \subset \eta_{d}=\lambda \\
\left|\eta_{i} / \eta_{i-1}\right|=a(b / d)}} \prod_{i=1}^{d}\left(\sum_{\substack{\operatorname{SSST} T \\
\operatorname{sh}(T)=\eta_{i} / \eta_{i-1}, \operatorname{wt}(T)=a^{(b / d)}}} \omega_{b / d}^{\operatorname{maj}(T)}\right)
\end{aligned}
$$

Proof: Let $T$ be a semistandard skew-tableau of shape $\lambda / \mu$ and weight $a^{b}$. Let $\eta_{i}$ be the shape formed by the entries of $T$ with values in $\{1, \ldots, i(b / d)\}$. Decompose $T$ into a sequence of $d$ semistandard skew-tableaux $\left(T_{1}, \ldots, T_{d}\right)$ by letting $T_{i}$ be the entries of $T$ in positions $\eta_{i} / \eta_{i-1}$ reduced by $(i-1)(b / d)$ so that the values run from 1 to $b / d$. For example, let $T$ be

$\begin{array}{lllll} & & & & 3 \\ 1 & 1 & 2 & 3 & \\ 2 & 3 & 4 & 4 & \end{array}$

with $a=3, b=4$, and $d=2$. Then

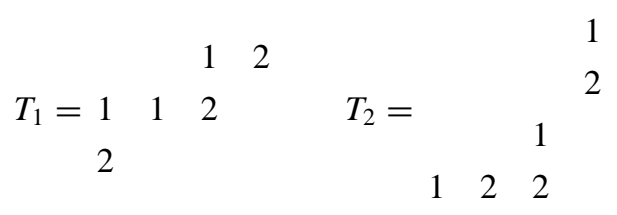

This gives a bijection between the terms appearing on the two sides of (2). It is clear that under this bijection

$$
\omega_{b}^{d \operatorname{maj}(T)}=\prod_{i} \omega_{b / d}^{\operatorname{maj}\left(T_{i}\right)}
$$

which shows that term for term the two sides of (2) are equal.

Theorem 7.5 Let $\lambda \vdash n=a b$ and $\mu \vdash b$. Take $\sigma \in S_{b}$ of cycle type $\mu$. Then the eigenvalues of $\sigma$ acting on $S^{\lambda, b}$ are $\left\{\underline{\omega}^{\text {maj }_{\mu}(T)}: T \in \mathcal{S}^{\lambda, b}\right\}$. 
Proof: Let $\sigma$ be a permutation of type $\mu$. Pick $k$. The cycle type of $\sigma^{k}$ is the partition which contains $\operatorname{gcd}\left(k, \mu_{i}\right)$ parts equal to $\mu_{i} / \operatorname{gcd}\left(k, \mu_{i}\right)$ for $1 \leq i \leq l(\mu)$. Call this partition $\mu_{*}$. Let $d_{i}=\operatorname{gcd}\left(k, \mu_{i}\right)$.

By Theorem 6.2 and Corollary 2.3,

$$
\begin{aligned}
& \chi^{\lambda, b}(\mu)=\sum_{\substack{\operatorname{SST} T \\
\operatorname{sh}(T)=\lambda \operatorname{wt}(T)=a^{b}}} \underline{\omega}^{\operatorname{maj}_{\mu}(T)} . \\
& \sum_{\substack{\operatorname{SST} T \\
\operatorname{sh}(T)=\lambda \operatorname{wt}(T)=a^{b}}}\left(\underline{\omega}^{\operatorname{maj}_{\mu}(T)}\right)^{k} \\
& =\sum_{\substack{\emptyset=\nu_{0} \subset \cdots \subset \nu_{l}=\lambda \\
\left|\nu_{i} / \nu_{i-1}\right|=\mu_{i}}} \prod_{i=1}^{l}\left(\sum_{\substack{\operatorname{SSST} T \\
\operatorname{sh}(T)=\nu_{i} / \nu_{i-1} \operatorname{wt}(T)=a^{\mu_{i}}}} \omega_{\mu_{i}}^{k \operatorname{maj}(T)}\right) \\
& =\sum_{\substack{\emptyset=\nu_{0} \subset \cdots \subset \nu_{l}=\lambda \\
\left|\nu_{i} / \nu_{i-1}\right|=\mu_{i}}} \prod_{i=1}^{l}\left(\sum_{\substack{\operatorname{SSST} T \\
\operatorname{sh}(T)=v_{i} / \nu_{i-1} \operatorname{wt}(T)=a^{\mu_{i}}}} \omega_{\mu_{i}}^{d_{i} \operatorname{maj}(T)}\right) \\
& =\sum_{\substack{\emptyset=\nu_{0} \subset \cdots \subset \nu_{l}=\lambda \\
\left|\nu_{i} / \nu_{i-1}\right|=\mu_{i}}} \prod_{i=1}^{l}\left(\sum_{\substack{v_{i-1}=\eta_{i, 0} \subset \cdots \subset \eta_{i, d_{i}}=v_{i} \\
\left|\eta_{i, j} / \eta_{i, j-1}\right|=a\left(\mu_{i} / d_{i}\right)}} \prod_{j=1}^{d_{i}}\left(\sum_{\begin{array}{c}
\operatorname{SSST} T \\
\operatorname{sh}(T)=\eta_{i, j} / \eta_{i, j-1} \\
\mathrm{wt}(T)=a^{\left(\mu_{i} / d_{i}\right)}
\end{array}} \omega_{\mu_{i} / d_{i}}^{\operatorname{maj}(T)}\right)\right) \\
& =\sum_{\substack{\emptyset=\eta_{1,0} \subset \cdots \subset \eta_{1, d_{1}} \\
=\eta_{2,0} \subset \cdots \subset \eta_{l, d_{l}}=\lambda}} \prod_{i=1}^{l} \prod_{j=1}^{d_{i}}\left(\sum_{\begin{array}{c}
\mathrm{SSST} T \\
\operatorname{sh}(T)=\eta_{i, j} / \eta_{i, j-1} \\
\operatorname{wt}(T)=a^{\left(\mu_{i} / d_{i}\right)}
\end{array}} \omega_{\mu_{i} / d_{i}}^{\operatorname{maj}(T)}\right) \\
& =\sum_{\substack{\operatorname{SST} T \\
\operatorname{sh}(T)=\lambda \operatorname{wt}(T)=a^{b}}} \underline{\omega}^{\text {maj }_{\mu_{*}}(T)} S_{\operatorname{sh}(T)}(\underline{x}) \\
& =\chi^{\lambda, b}\left(\mu_{*}\right)
\end{aligned}
$$

So, by Proposition 7.1, the result is proven.

When $\lambda \vdash b$, Theorem 7.5 reduces to the result of J. Stembridge [11] on the eigenvalues of the action of permutations on the irreducible representations of the symmetric group.

\section{Appendix A: Extended Example}

Example 11 The charts below give $\underline{\omega}^{\text {maj }_{\mu}(T)}$ for all $\mu \vdash 3$ and all semistandard tableaux $T$ of weight $2^{3}$. The charts are organized based on the shape $\lambda$ of $T$. The column sums are the character values $\chi^{\lambda, 3}$. 
$\lambda=(6)$

\begin{tabular}{|cccccc|ccc|}
\hline \multicolumn{4}{|c|}{$T$} & & $\underline{\omega}^{\text {maj }_{(1,1,1)}(T)}$ & $\underline{\omega}^{\text {maj }_{(2,1)}(T)}$ & $\underline{\omega}^{\text {maj }_{(3)}(T)}$ \\
\hline 1 & 1 & 2 & 2 & 3 & 3 & 1 & 1 & 1 \\
\hline & & & & & 1 & 1 & 1 \\
\hline
\end{tabular}

$\lambda=(5,1)$

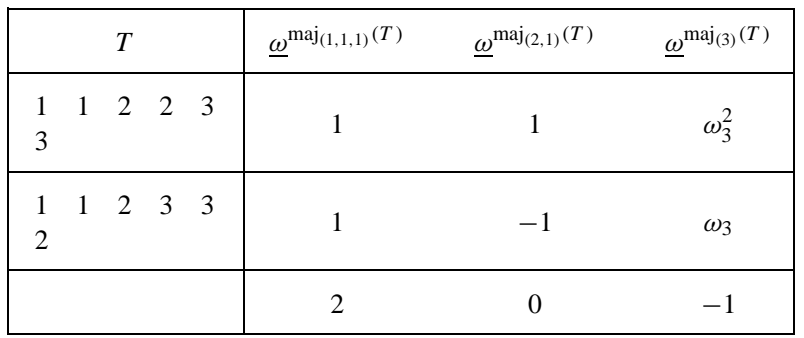

$\lambda=(4,2)$

\begin{tabular}{|c|c|c|c|c|c|}
\hline \multicolumn{3}{|c|}{$T$} & $\underline{\omega}^{\operatorname{maj}_{(1,1,1)}(T)}$ & $\underline{\omega}^{\operatorname{maj}_{(2,1)}(T)}$ & $\underline{\omega}^{\operatorname{maj}_{(3)}(T)}$ \\
\hline $\begin{array}{l}1 \\
3\end{array}$ & $\begin{array}{l}1 \\
3\end{array}$ & 2 & 1 & 1 & $\omega_{3}$ \\
\hline $\begin{array}{l}1 \\
2\end{array}$ & $\begin{array}{l}1 \\
3\end{array}$ & 2 & 1 & -1 & 1 \\
\hline \multirow[t]{2}{*}{$\begin{array}{l}1 \\
2\end{array}$} & $\begin{array}{l}1 \\
2\end{array}$ & 3 & 1 & 1 & $\omega_{3}^{2}$ \\
\hline & & & 3 & 1 & 0 \\
\hline
\end{tabular}

$\lambda=(4,1,1)$

\begin{tabular}{|ccccccc|}
\hline \multicolumn{3}{|c|}{$T$} & $\underline{\omega}^{\text {maj }_{(1,1,1)}(T)}$ & $\underline{\omega}^{\operatorname{maj}_{(2,1)}(T)}$ & $\underline{\omega}^{\operatorname{maj}_{(3)}(T)}$ \\
\hline 1 & 1 & 2 & 3 & & -1 & 1 \\
3 & & & 1 & -1 & 1 \\
\hline & & & 1 & & \\
\hline
\end{tabular}

$\lambda=(3,3)$

\begin{tabular}{|ccc|ccc|}
\hline \multicolumn{2}{|c|}{$T$} & $\underline{\omega}^{\text {maj }_{(1,1,1)}(T)}$ & $\underline{\omega}^{\operatorname{maj}_{(2,1)}(T)}$ & $\underline{\omega}^{\operatorname{maj}_{(3)}(T)}$ \\
\hline 1 & 1 & 2 & 1 & -1 & 1 \\
2 & 3 & 3 & & -1 & 1 \\
\hline & & 1 & -1 \\
\hline
\end{tabular}


$\lambda=(3,2,1)$

\begin{tabular}{|c|c|c|c|c|}
\hline & $T$ & $\underline{\omega}^{\operatorname{maj}_{(1,1,1)}(T)}$ & $\underline{\omega}^{\operatorname{maj}_{(2,1)}(T)}$ & $\underline{\omega}^{\mathrm{maj}_{(3)}(T)}$ \\
\hline $\begin{array}{l}1 \\
2 \\
3\end{array}$ & $\begin{array}{ll}1 & 2 \\
3 & \end{array}$ & 1 & -1 & $\omega_{3}^{2}$ \\
\hline \multirow[t]{2}{*}{$\begin{array}{l}1 \\
2 \\
3\end{array}$} & $\begin{array}{ll}1 & 3 \\
2 & \end{array}$ & 1 & 1 & $\omega_{3}$ \\
\hline & & 2 & 0 & -1 \\
\hline
\end{tabular}

$\lambda=(2,2,2)$

\begin{tabular}{|c|c|c|c|}
\hline$T$ & $\underline{\omega}^{\operatorname{maj}_{(1,1,1)}(T)}$ & $\underline{\omega}^{\operatorname{maj}_{(2,1)}(T)}$ & $\underline{\omega}^{\operatorname{maj}_{(3)}(T)}$ \\
\hline $\begin{array}{ll}1 & 1\end{array}$ & \multirow{3}{*}{1} & \multirow{3}{*}{1} & \multirow{3}{*}{1} \\
\hline 22 & & & \\
\hline $\begin{array}{ll}3 & 3\end{array}$ & & & \\
\hline & 1 & 1 & 1 \\
\hline
\end{tabular}

\section{Acknowledgments}

I would like to thank R. Stanley for some discussions and for pointing out Gay's paper to me. J. Stembridge's Maple package SF for doing symmetric function computations was used extensively in the research leading up to this paper. In particular, I would not have discovered Theorem 2.2 without the use of this package. I appreciate J.-Y. Thibon pointing out the results in [6] which greatly simplified the proof of Theorem 2.2. Lastly, I would like to thank the anonymous referee for numerous suggestions and corrections. The referee also pointed out that the statistic maj $(T)$ of this paper is related to the statistic $d(T)$ in [7] when $\operatorname{wt}(T)=b^{a}$. I was not able to use this to simplify the paper.

\section{References}

1. L. Butler, "Subgroup lattices and symmetric functions," Memoirs of the Amer. Math. Soc. 539 (1994), Amer. Math. Soc., Providence, RI.

2. Y. Chen, A. Garsia, and J. Remmel, "Algorithms for plethysm," Contemporary Mathematics 34 (1984), 109-153.

3. D. Gay, "Characters of the Weyl group of $S U(n)$ on zero weight spaces and centralizers of permutation representations," Rocky Moun. J. Math. 6 (1976), 449-455.

4. G. James, The Representation Theory of the Symmetric Group, Lecture Notes in Mathematics 682, SpringerVerlag, Berlin/Heidelberg/New York, 1978.

5. G. James and A. Kerber, The Representation Theory of the Symmetric Group, Encyclopedia of Mathematics, Vol. 16, Addison-Wesley, Reading, MA, 1981.

6. A. Lascoux, B. Leclerc, and J.-Y. Thibon, "Green polynomials and Hall-Littlewood functions at roots of unity," European J. Combinatorics 15 (1994), 173-180.

7. A. Lascoux, B. Leclerc, and J.-Y. Thibon, "Crystal graphs and $q$-analogues of weight multiplicities for the root system $A_{n}$," Letters Math. Phys. 35 (1995), 359-374. 
8. A. Lascoux and M. Schützenberger, "Sur une conjecture de H.O. Foulkes," C.R. Acad. Sci. Paris 286A (1978), 323-324.

9. I. Macdonald, Symmetric Functions and Hall Polynomials, 2nd edition, Clarendon Press, Oxford, 1995.

10. B. Sagan, The Symmetric Group: Representations, Combinatorial Algorithms, and Symmetric Functions, Wadsworth \& Brooks/Cole, Pacific Grove, CA, 1991.

11. J. Stembridge, "On the eigenvalues of representations of reflection groups and wreath products," Pac. J. Math. 140 (1989), 353-395. 